\title{
Comparison of the Changes Following Two Treatment Approaches: Rapid Maxillary Expansion Versus Alternate Rapid Maxillary Expansion and Constriction
}

\author{
Fatih Çelebi' (D), Murat Çelikdelen² (D) \\ 'Department of Orthodontics, Tokat Gaziosmanpasa University School of Dentistry, Tokat, Turkey \\ 2Private Practice, Tokat, Turkey
}

Cite this article as: Çelebi F, Çelikdelen M. Comparison of the Changes Following Two Treatment Approaches: Rapid Maxillary Expansion Versus Alternate Rapid Maxillary Expansion and Constriction. Turk J Orthod 2020; 33(1): 1-7.

\begin{abstract}
Objective: The aim of the present study was to evaluate the hard and soft tissue changes following rapid maxillary expansion (RME) and alternate rapid maxillary expansion and constriction (Alt-RAMEC) therapies.

Methods: A total of 54 patients who needed maxillary expansion or Alt-RAMEC procedure were recruited and divided into two groups (27 subjects in the RME group and 27 subjects in the Alt-RAMEC group). Expansion screw was activated $0.5 \mathrm{~mm} /$ day ( 2 turns/day) in the RME group. Approximately $11 \mathrm{~mm}$ of expansion was achieved. In the Alt-RAMEC group, the screw was activated $1 \mathrm{~mm} /$ day (4 turns/day) during a period of 4 weeks. In the first and third weeks, the screw was opened; in the second and fourth weeks, the screw was closed. Cephalometric tracing and analyzing were done with the aid of digital software. Lateral cephalometric radiographs were obtained before (T0) and after (T1) RME and Alt-RAMEC applications.

Results: In the RME group, the maxilla moved forward and downward. Upper incisor retrusion was observed according to the reference planes. In addition, the tip of the nose moved forward, and the upper lip moved downward. In the Alt-RAMEC group, the nasolabial angle became more obtuse, and the stomion superius moved backward and downward.

Conclusion: RME therapy resulted in skeletal and dental changes in the maxilla and related structures, favoring a contribution to solving Class III problems. No remarkable changes were recorded in the Alt-RAMEC group.
\end{abstract}

Keywords: RME, Alt-RAMEC, Class III

\section{INTRODUCTION}

Rapid maxillary expansion (RME) is a traditional method for correcting transverse maxillary deficiency. Although its popularity has changed over time, RME has become a fundamental part of dentofacial orthopedics in modern orthodontics. The maxilla is subjected to heavy forces that can create orthopedic effect, and transverse deficiency problem is solved by separating the maxillary halves. The qualification of the obtained effect varies according to several factors, such as the maturational stage, appliance design, and treatment protocol.

In addition to the treatment of transverse deficiency, RME has been recommended for Class III patients in combination with face mask therapy. The rationale behind this combined treatment is that it disarticulates circummaxillary and intermaxillary sutures, which facilitates maxillary protraction $(1,2)$. Because the maxilla is loosened from the craniofacial complex, the orthopedic traction of a face mask can be more efficient than that of a face mask therapy alone. Although the amount of advancement varies in different studies, it has been demonstrated that 1.5-3 mm maxillary advancement could be achieved with RME-face mask therapy $(3,4)$. 
When there is no transverse maxillary deficiency, the degree of expansion is a controversial issue in the literature. Alcan et al. (5) expanded the maxilla for 5 days with a 2 turns/day protocol before protraction. Other authors have also reported that the duration of expansion must be limited to $7-10$ days $(4,6,7)$. However, Haas (8) advocated that at least 12-15 mm expansion must be achieved for the disarticulation of the circummaxillary and intermaxillary sutures before face mask therapy. It is apparent that if there is no transverse deficiency, 12-15 mm expansion for disarticulation is too much with respect to the correction by the compensation mechanism.

In 2005, Liou (9) introduced a new method called alternate RMEs and constrictions (Alt-RAMEC). In this method, the maxilla is expanded $1 \mathrm{~mm} /$ day for the first week and then constricted $1 \mathrm{~mm} /$ day for the following week with the aid of a two-hinged expander. This protocol is repeated for 7-9 weeks (9-11). It was advocated that the Alt-RAMEC procedure is a better option than RME. While the maxilla is moved forward approximately $1.5-3 \mathrm{~mm}$ with RMEface mask treatment $(3,4)$, Liou and Tsai (9) showed that a $5.8 \mathrm{~mm}$ maxillary advancement can be achieved at the level of A point by maxillary protraction combined with Alt-RAMEC. In the study by Isci et al. (12), $2.33 \mathrm{~mm}$ of maxillary movement was obtained in the RME group, and $4.13 \mathrm{~mm}$ of anterior movement was achieved in the Alt-RAMEC group, with an approximately two-fold movement.

Many researchers have investigated the effects of RME on dentofacial structure, but there are conflicting results with respect to sagittal and vertical changes at the maxilla. Chung and Font stated that maxillary forward and downward movements are induced by expansion. Additionally, the mandible moved downward and backward, and the anterior facial height increased (13). Baratieri et al. (14) studied dental and skeletal changes due to RME in Class II, Division 1 patients and reported immediat maxillary forward movement after therapy. However, in a systematic review, Lagravere et al. (15) investigated the long-term skeletal changes in patients undergoing RME therapy and concluded that RME does not produce significant sagittal and vertical changes at the position of the maxilla. Da Silva Filho et al. (16) researched the short-term results for RME and noted no significant anterior displacement of the maxilla.

Since Alt-RAMEC is a relatively new method, the studies are limited. There are even fewer articles available on the use of Alt-RAMEC in addition to face mask therapy. Yilmaz and Kucukkeles (17) investigated skeletal, soft tissue, and airway changes following Alt-RAMEC protocol. They concluded that a slight forward $(0.89 \pm 0.93 \mathrm{~mm})$ and downward $(0.92 \pm 1.62 \mathrm{~mm})$ movement of the maxilla occurred following the Alt-RAMEC procedure. A slight improvement of the overjet was recorded in all Class III subjects.

Researches in this area were generally focused on the total outcomes of combined therapy (Alt-RAMEC or RME-face mask), and the effects of the second part of the combined therapy (Alt-RAMEC or RME) were neglected. The increased effectiveness of combined therapy (Alt-RAMEC or RME-face mask) in comparison with single therapy (face mask) can be attributed to sutural mobilization or the direct skeletal effects of the second part of therapy (Alt-RAMEC or RME). This must be clarified. The aims of the present study were to evaluate and compare the hard and soft tissue changes following RME and Alt-RAMEC.

\section{METHODS}

This prospective study was reviewed and approved by the Clinical Research Ethics Committee of the Tokat Gaziosmanpaşa University. A total of 54 patients who needed transverse maxillary expansion or an Alt-RAMEC procedure, according to the treatment plan, were recruited in the study. The patients were divided into two groups: 27 subjects in the RME group and 27 subjects in the Alt-RAMEC group. For both groups, tooth- and tissue-borne rapid maxillary expanders were used (Figure 1).

The RME group consisted of 18 female and 9 male subjects. The mean age of the RME group was $12.5 \pm 1.9$ years. Expansion screws were activated $0.5 \mathrm{~mm} /$ day ( 2 turns/day). The average expansion at the screw level was $11 \mathrm{~mm}$.

The Alt-RAMEC group consisted of 17 female and 10 male subjects. The mean age of the Alt-RAMEC group was $12.8 \pm 1.6$ years. Parents were instructed to activate the screw $1 \mathrm{~mm} /$ day (4 turns/ day). In the first and third weeks, the screw was opened; in the second and fourth weeks, the screw was closed. At the end of the 4-week Alt-RAMEC procedure, the clinician controlled whether or not the screw was at a closed position. Subjects who did not achieve screw activation were excluded from the study.

Lateral cephalometric radiographs were obtained before (T0) and after (T1) RME and Alt-RAMEC applications. After completing the RME and Alt-RAMEC applications, orthodontic treatments were continued in accordance with the predetermined treatment plan. Informed consent was obtained from the parents of the participants.

\section{Cephalometric Analysis}

Cephalograms were traced and analyzed with the aid of the Dolphin software (ver. 11.5; Dolphin Imaging and Management Solutions,

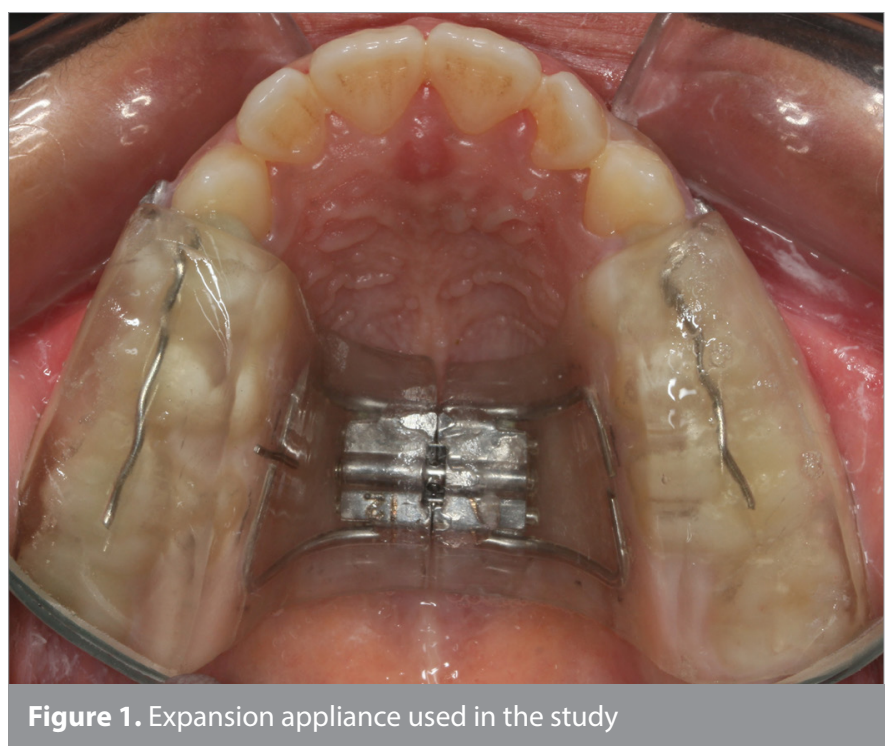




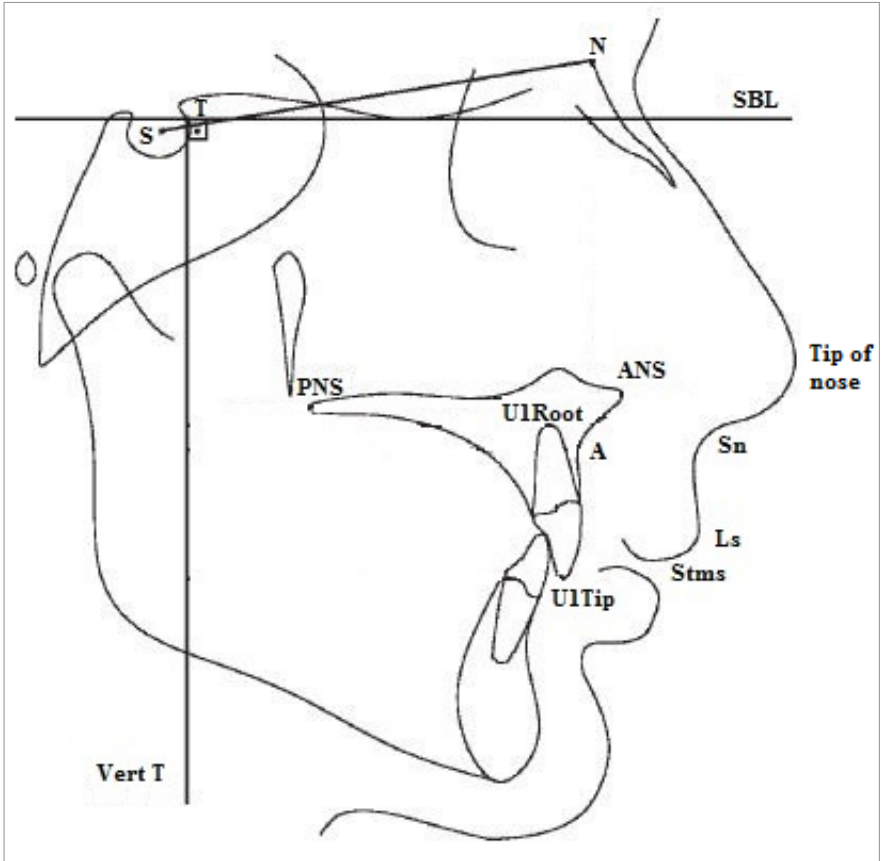

Figure 2. Cephalometric landmarks

Sella (S): Center of the pituitary fossa. Nasion (N): The most anterior point of the

frontonasal suture in the median plane. Point $A(A)$ : Deepest point on the curve of the maxilla between the anterior nasal spine and supradentale. Anterior nasal spine (ANS): Tip of the anterior nasal spine. Posterior nasal spine (PNS): Tip of the posterior nasal spine. Point T (T): Most superior point of the anterior wall of the sella turcica at the junction of the tuberculum sellae. U1Tip: Tip of the upper central teeth. U1Root: Root of the upper central teeth. Subnasale (Sn): Base of the columella that meets the upper lip. Labrale superior (Ls): Vermillion border of the upper lip in the midsagittal plane. Stomion superius (Stms): Lowest midline point of the upper lip

Chatsworth, CA, USA) by the same operator. Lateral cephalometric radiographs were obtained before (TO) and after (T1) RME and Alt-RAMEC applications. Hard and soft tissue landmarks belonging to the mandible were not used in the present study. The cephalometric landmarks utilized in the present study are shown in Figure 2.

Horizontal and vertical basic reference planes that were utilized for measurements were the following (Figure 2):

1. Stable basicranial line (SBL): horizontal line that passes through the most superior point of the anterior wall of the sella turcica at the junction with the tuberculum sellae (point $\mathrm{T}$ ), and it is tangent to the lamina cribrosa of the ethmoid. It was stated that these structures are stable in the rest of life after from the age of 4-5 years (18).

2. Vertical $T($ Vert $T)$ : a line passing through point $T$ and perpendicular to SBL.

The other measurements used in the present study were the following:

1. SNA angle: angle constructed by the intersection of the nasion-sella and nasion-point $A$ lines

2. SBL-PP angle: angle constructed by the intersection of the stable basicranial line and the palatal plane

3. U1-PP angle: angle constructed by the intersection of the long axis of the maxillary central incisor and the palatal plane

4. U1-SBL angle: angle constructed by the intersection of the long axis of the maxillary central incisor and the stable basicranial line
5. Nasolabial angle: angle constructed by the intersection of a line passing from the subnasale and tangent to the lower border of the nose with a line from the labrale superius to the subnasale

6. Vert T-ANS: perpendicular distance from the anterior nasal spine (ANS) to the vertical reference line

7. Vert T-PNS: perpendicular distance from the posterior nasal spine (PNS) to the vertical reference line

8. Vert T-A: perpendicular distance from point $A$ to the vertical reference line

9. Vert T-U1Tip: perpendicular distance from the incisal edge of the maxillary central incisor to the vertical reference line

10. Vert T-U1Root: perpendicular distance from the maxillary central incisor root apex to the vertical reference line

11. ANS-SBL: perpendicular distance from the ANS to the stable basicranial line

12. PNS-SBL: perpendicular distance from the PNS to the stable basicranial line

13. A-SBL: perpendicular distance from point $A$ to the stable basicranial line

14. SBL-U1Tip: perpendicular distance from the incisal edge of the maxillary central incisor to the stable basicranial line

15. SBL-U1Root: perpendicular distance from the maxillary central incisor root apex to the stable basicranial line

16. A-Nperp: perpendicular distance from point $A$ to the nasion perpendicular line

17. Vert T-Tip of Nose: perpendicular distance from the tip of the nose to the vertical reference line

18. Vert T-Sn: perpendicular distance from the subnasale to the vertical reference line

19. Vert T-Ls: perpendicular distance from the labrale superior to the vertical reference line

20. Vert T-Stms: perpendicular distance from the stomion superius to the vertical reference line

21. SBL-Tip of Nose: perpendicular distance from the tip of the nose to the stable basicranial line

22. SBL-Sn: perpendicular distance from the subnasale to the stable basicranial line

23. SBL-Ls: perpendicular distance from the labrale superior to the stable basicranial line

24. SBL-Stms: perpendicular distance from the stomion superius to the stable basicranial line.

\section{Statistical Analysis}

Statistical analysis was performed using the Statistical Package for the Social Sciences for Windows, version 19.0 (IBM Corp., Armonk, NY, USA). Chi-square test was used to assess gender difference between the groups. At the beginning of the treatments, independent samples t-test was used for comparisons of age and pretreatment cephalometric data among the groups. Independent samples t-test and paired samples t-test were used to evaluate and compare the post-treatment changes among the groups. A $p$ value of $<0.05$ was considered statistically significant.

\section{RESULTS}

There were no differences with respect to gender (RME: 18 female and 9 male and Alt-RAMEC: 17 female and 10 male, $p=0.776$ ) or age 


\begin{tabular}{|c|c|c|c|}
\hline & \multicolumn{2}{|c|}{ Groups } & \multirow[b]{2}{*}{$\mathbf{p}$} \\
\hline & $\begin{array}{c}\text { RME } \\
\text { Mean } \pm \text { SD }\end{array}$ & $\begin{array}{l}\text { Alt-RAMEC } \\
\text { Mean } \pm \text { SD }\end{array}$ & \\
\hline SNA $\left({ }^{\circ}\right)$ & $79.91 \pm 3.42$ & $79.67 \pm 3.34$ & 0.792 \\
\hline SBL-PP $\left({ }^{\circ}\right)$ & $1.25 \pm 4.76$ & $1.22 \pm 3.62$ & 0.980 \\
\hline U1-PP $\left(^{\circ}\right)$ & $112.95 \pm 8.87$ & $116.23 \pm 4.04$ & 0.086 \\
\hline U1-SBL $\left({ }^{\circ}\right)$ & $111.67 \pm 9.49$ & $114.98 \pm 4.24$ & 0.104 \\
\hline Nasolabial angle $\left({ }^{\circ}\right)$ & $107.62 \pm 8.11$ & $108.43 \pm 10.09$ & 0.746 \\
\hline Vert T-ANS (mm) & $63.34 \pm 4.61$ & $62.27 \pm 4.97$ & 0.416 \\
\hline Vert T-PNS (mm) & $13.29 \pm 3.91$ & $14.5 \pm 3.61$ & 0.241 \\
\hline Vert T-A (mm) & $58.19 \pm 4.57$ & $57.49 \pm 4.65$ & 0.582 \\
\hline Vert T-U1Tip (mm) & $59.65 \pm 7.07$ & $60.24 \pm 5$ & 0.724 \\
\hline Vert T-U1Root (mm) & $51.09 \pm 4.9$ & $50.61 \pm 4.2$ & 0.706 \\
\hline ANS-SBL (mm) & $43.6 \pm 4.76$ & $42.84 \pm 3.35$ & 0.501 \\
\hline PNS-SBL (mm) & $42.51 \pm 3.01$ & $41.81 \pm 3.78$ & 0.453 \\
\hline A-SBL $(\mathrm{mm})$ & $46.84 \pm 4.05$ & $46.22 \pm 3.4$ & 0.549 \\
\hline SBL-U1Tip (mm) & $69.37 \pm 4.81$ & $68.37 \pm 4.82$ & 0.450 \\
\hline SBL-U1Root (mm) & $47.62 \pm 4.66$ & $47.69 \pm 4.15$ & 0.951 \\
\hline A-Nperp (mm) & $-1.09 \pm 4.3$ & $-0.66 \pm 2.94$ & 0.673 \\
\hline Vert T-Tip of Nose (mm) & $85.02 \pm 5.4$ & $83.97 \pm 5.71$ & 0.490 \\
\hline Vert T-Sn (mm) & $72.46 \pm 5.27$ & $71.41 \pm 4.97$ & 0.457 \\
\hline Vert T-Ls (mm) & $73.32 \pm 6.27$ & $72.8 \pm 5.45$ & 0.744 \\
\hline Vert T-Stms (mm) & $66.95 \pm 6.41$ & $67.23 \pm 5.26$ & 0.861 \\
\hline SBL-Tip of Nose $(\mathrm{mm})$ & $39.24 \pm 5.97$ & $38.64 \pm 5.37$ & 0.699 \\
\hline SBL-Sn (mm) & $47.31 \pm 5.41$ & $47.5 \pm 4.92$ & 0.894 \\
\hline SBL-Ls (mm) & $60.96 \pm 5.49$ & $61.19 \pm 5.34$ & 0.879 \\
\hline SBL-Stms (mm) & $66.17 \pm 5.55$ & $65.99 \pm 5.18$ & 0.902 \\
\hline
\end{tabular}

(RME: $12.55 \pm 1.94$ years and Alt-RAMEC: $12.88 \pm 1.68$ years, $p=0.505$ ) in the subjects. The groups did not show any differences with respect to pretreatment cephalometric measurements (Table 1).

As a result of treatment in the RME group, changes in measurements of SNA, U1-PP, U1-SBL, Vert T-ANS, Vert T-A, Vert TU1Root, ANS-SBL, PNS-SBL, A-SBL, SBL-U1Root, Vert T-Tip of Nose, and SBL-Ls were significantly different. SNA, Vert T-ANS, Vert T-A, Vert T-U1Root, ANS-SBL, PNS-SBL, A-SBL, SBL-U1Root, Vert T-Tip of Nose, and SBL-Ls increased significantly, whereas changes in measurements of U1-PP and U1-SBL decreased significantly. The maxilla moved forward and downward without a change in the palatal plane. Significant upper incisor retrusion was found according to both palatal plane and basicranial line. In addition, the tip of the nose moved forward, and the upper lip moved downward (Table 2).

In the Alt-RAMEC group, changes in nasolabial angle, Vert T-Stms, SBL-Ls, and SBL-Stms were statistically significant. Nasolabial angle, SBL-Ls, and SBL-Stms increased significantly, and Vert T-Stms decreased significantly. The nasolabial angle became more obtuse, and the stomion superius moved backward and downward. In addition, the labrale superior moved downward (Table 2).

\section{DISCUSSION}

The Alt-RAMEC procedure was first introduced in patients with cleft lip and palate to enhance the Class III therapy by Liou and Tsai (9). Instead of using a Haas/Hyrax expander, they employed a double-hinged expander to achieve a more effective expansion in the anterior region (19). They asserted that this type of expander could provide more forward displacement of the maxilla without the possibility of bone resorption behind the maxillary tuberosity. The original weekly sequence of Alt-RAMEC was 9 weeks, including four pairings of expansion-constriction, followed by a final expansion. However, it has been modified and used differently in many studies. For instance, Canturk and Celikoglu (20) used the protocol along 8 weeks without the final expansion week. Similarly, Isci et al. (12) modified the protocol, and it was 4 weeks in their study. There was no final expansion week in their study, as well. Maino et al. (21) used a 5-week protocol in their study. For instance, Isci et al. (12) and Canturk and Celikoglu (20) used a Haas/ Hyrax-type expander in their studies. Maino et al. (21) used a Haas/ Hyrax-type expander with mini-screw and tooth anchorage. Similarly, several researchers have selected to apply different weekly sequences without reporting any reason: 9-, 8-, 5-, and 4-weekly sequences have been used $(12,17,20,21)$. There is no clear consensus about which expander and procedure must be utilized.

In the present study, the classical type of expander was used. In patients with cleft palate, mostly, there is dentoalveolar developmental failure in the anterior region due to tooth agenesis or unerupted teeth, so the use of a double-hinged expander or fan-type expander, which can provide further expansion in the anterior segment, is reasonable. However, our study involved subjects who had no cleft lip and palate, so there was no need for extra expansion at the anterior segment. Furthermore, although it has been claimed that a double-hinged expander has an advantage in maxillary protraction compared with Haas/Hyrax-type, no clear evidence has been presented to support this view, except for schematic drawings by their proponents.

A 4-weekly sequence was used without a final expansion week in this study, and screw activation was $1 \mathrm{~mm} /$ day (4 turns/day). We preferred to terminate the activation procedure at the initial screw position. Because the Alt-RAMEC procedure has been first introduced as an alternative to the RME procedure and it has been claimed that it does not require unnecessary maxillary expansion in Class III therapy (9). Whereas, if we had added the final expansion week to our procedure, we would have achieved a $7 \mathrm{~mm}$ expansion. $7 \mathrm{~mm}$ of expansion is almost half of a full screw activation. Since we have thought that such an expansion was contrary to the emergence claim of Alt-RAMEC, we did not include the 5th week (expansion week) in the activation procedure.

In the present study, pure treatment outcomes of RME and of Alt-RAMEC (without any combined therapy) were evaluated and compared. Previous studies have shown that when these treatments are used together with a face mask, the skeletal advancement in the sagittal direction can be greater than that achieved by face mask therapy alone. There are many RME studies in the literature. Several studies have examined RME's contribution to treatments of Class III malocclusion, and two theories have been 
Table 2. Changes in RME and Alt-RAMEC groups during treatment

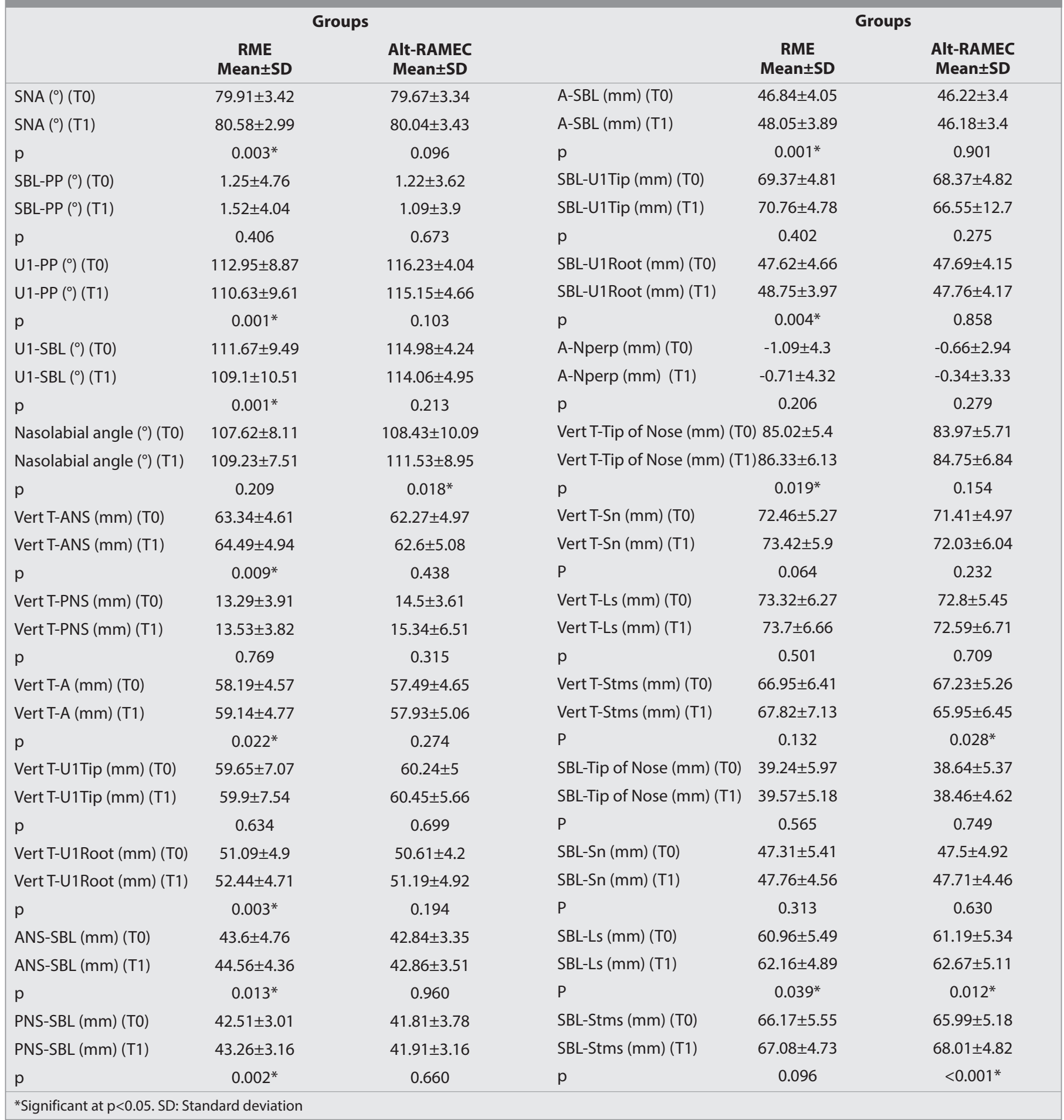

presented. In the first theory, without a face mask, it has been claimed that the maxilla slightly moves forward with the aid of RME. However, some studies have shown that the maxilla goes in a backward direction rather than forward as a result of RME. In this regard, this issue is controversial. In the second theory, it was stated that sutural mobilization caused by RME may increase the efficiency of face mask. This is also a matter of debate because sutural mobilization is not irrational, but it is not a measurable phenomenon, except for theoretical models and animal studies.
In contrast, research investigating Alt-RAMEC is relatively limited. Available studies are mostly about the efficacy of combined therapies. We could identify only two studies that focused on the effects of using Alt-RAMEC alone $(9,17)$. Liou and Tsai (9) examined whether Alt-RAMEC is effective in solving Class III malocclusion. They tested the hypothesis that Alt-RAMEC displaces the maxilla more anteriorly and disarticulates circummaxillary sutures more effectively than RME. However, the participants in their study had unilateral cleft lip and palate, so evaluating the 
effectiveness of Alt-RAMEC in Class III patients without cleft lip and palate may reveal different results. The anatomies of patients with cleft lip and palate are different from those of normal subjects, so treatment efficiency may differ accordingly. In the other study by Yilmaz and Kucukkeles (17), changes were evaluated following the Alt-RAMEC protocol using cone beam computed tomography. We could not find any other isolated Alt-RAMEC studies.

According to our results, the maxilla moved forward due to RME (SNA, Vert T-A, and Vert T-ANS increased at $0.67^{\circ}, 0.95 \mathrm{~mm}$, and $1.15 \mathrm{~mm}$, respectively). This is consistent with the previous findings reported by Haas, Davis and Kronman, and Chung and Font, and others $(1,2,13,22-26)$. However, there are also contradictory results in the literature. Da Silva Filho et al. (16) stated that the anterior displacement of the maxilla with significant changes in the SNA angle should not be expected. Similarly, Sarver and Johnston (27) showed that bonded RME causes backward displacement, as opposed to forward movement. We think that many factors, such as appliance design, the subjects' maturation stage, and expansion protocol, could explain these contradictory results. Biederman (25) schematically showed how the maxilla can move in the sagittal direction according to the location of the center of rotation. When the maxilla is expanded and there is no resorption at the site of the bony complex with which it articulates posteriorly, it leads to forward displacement due to the support from the posterior regions. Such a circumstance may have occurred in the present study.

Based on our results, we anticipate that RME can be used alone for mild maxillary retrusion in Class III subjects, as suggested by Haas (2). Studies conducted by Sung and Baik (28), Cha (29), Kapust et al. (30), and Isci et al. (12) showed $1.7 \mathrm{~mm}, 0.97 \mathrm{~mm}, 1.97$ $\mathrm{mm}$, and $2.33 \mathrm{~mm}$ of point $\mathrm{A}$ anterior movement, respectively, for subjects aged between 10-14 years, treated with RME and face mask. In our study, using RME alone resulted in a 0.95 $\mathrm{mm}$ anterior movement of point $A$. This effect is almost half the amount of that was obtained by RME and a face mask therapy. RME increased the SNA by $0.67^{\circ}$; it could be speculated that this amount of increase could not solve mild maxillary retrusion. However, it should not be overlooked that the maxilla moved not only forward but also downward (the increases of ANS-SBL, PNS-SBL, and A-SBL were statistically significant with $0.96 \mathrm{~mm}$, $0.75 \mathrm{~mm}$, and $1.21 \mathrm{~mm}$, respectively). Downward movement masks the angular changes in forward movement. Therefore, we think that Vert T-A, rather than SNA, will give more accurate information on the detection of RME's contribution to maxillary advancement.

Another finding is the downward movement of the maxilla (Table 2). This is not surprising when previous publications in the literature are considered. Since Haas' publications in the 1960s, many researchers have observed a downward movement due to RME $(1,2,13,16,26)$. Maxillary downward movement orients the mandible to a downward and backward direction, which results in a smaller SNB, higher mandibular plane angle, and longer anterior facial height. This outcome, just as forward movement, helps to resolve the sagittal discrepancy in Class III subjects.
In the Alt-RAMEC group, hard and soft tissues did not change to the same extent as they did in the RME group. Changes in nasolabial angle, Vert T-Stms, SBL-Ls, and SBL-Stms, which are all soft tissue parameters, were statistically significant. The nasolabial angle became more obtuse, and the upper lip moved backward and downward. Unfortunately, no adequate preliminary studies exist in the literature to help explain these data. Depending on the premature contacts that occurred after the Alt-RAMEC, the lips may have been incompetent, and these changes may have occurred when the subjects attempted to close their lips. In the RME group, the reason why these changes did not occur (except SBL-Ls) could be that the observed skeletal changes compensated for the soft tissue changes. In response to the backward movement of the upper lip, the maxilla moved forward and compensated the condition.

\section{CONCLUSION}

- $\quad$ RME therapy resulted in skeletal and dental changes in the maxilla and related structures, contributing to solve Class III problems. The maxilla moved forward and downward.

- No remarkable changes were recorded in the Alt-RAMEC group.

- In cases of mild maxillary retrusion accompanied by transverse deficiency, RME alone can be advised as a treatment.

Ethics Committee Approval: Ethics committee approval was received for this study from the Ethics Committee of Tokat Gaziosmanpaşa University.

Informed Consent: Written informed consent was obtained from the patients who participated in this study.

Peer-review: Externally peer-reviewed.

Author Contributions: Concept - F.C.; Design - F.C., M.Ç.; Supervision - F.C., M.Ç.; Data Collection and/or Processing - M.Ç.; Analysis and/or Interpretation - F.C., M.Ç.; Writing Manuscript - F.C.; Critical Reviews - F.C.

Conflict of Interest: The authors have no conflict of interest to declare.

Financial Disclosure: The authors declared that this study has received no financial support.

\section{REFERENCES}

1. Haas AJ. The treatment of maxillary deficiency by opening the midpalatal suture. Angle Orthod 1965; 35: 200-17.

2. Haas AJ. Palatal expansion: just the beginning of dentofacial orthopedics. Am J Orthod 1970; 57: 219-55. [CrossRef]

3. Williams MD, Sarver DM, Sadowsky PL, Bradley E. Combined rapid maxillary expansion and protraction facemask in the treatment of Class III malocclusions in growing children: A prospective longterm study. Semin Orthod 1997; 3: 265-74. [CrossRef]

4. Ngan P, Yiu C, Hu A, Hagg U, Wei SH, Gunel E. Cephalometric and occlusal changes following maxillary expansion and protraction. Eur J Orthod 1998; 20: 237-54. [CrossRef]

5. Alcan T, Keles A, Erverdi N. The effects of a modified protraction headgear on maxilla. Am J Orthod Dentofacial Orthop 2000; 117: 27-38. [CrossRef]

6. Baccetti T, McGill JS, Franchi L, McNamara JA Jr, Tollaro I. Skeletal effects of early treatment of Class III malocclusion with maxillary 
expansion and face-mask therapy. Am J Orthod Dentofacial Orthop 1998; 113: 333-43. [CrossRef]

7. Turley PK. Orthopedic correction of Class III malocclusion: retention and phase II therapy. J Clin Orthod 1996; 30: 313-24.

8. Haas AJ. The non-surgical treatment of the skeletal Class III. In: Book of Abstract. American Association of Orthodontists. 100th Annual Session; Chicago, III, April 29-May 3, 2000; 85.

9. Liou EJ, Tsai WC. A new protocol for maxillary protraction in cleft patients: repetitive weekly protocol of alternate rapid maxillary expansions and constrictions. Cleft Palate Craniofac J 2005; 42: 121-7. [CrossRef]

10. Liou EJ. Effective maxillary orthopedic protraction for growing Class III patients: a clinical application simulates distraction osteogenesis. Prog Orthod 2005; 6: 154-71.

11. Liou EJ. Toothborne orthopedic maxillary protraction in Class III patients. J Clin Orthod 2005; 39: 68-75.

12. Isci $D$, Turk T, Elekdag Turk S. Activation-deactivation rapid palatal expansion and reverse headgear in Class III cases. Eur J Orthod 2010; 32: 706-15. [CrossRef]

13. Chung $\mathrm{CH}$, Font $\mathrm{B}$. Skeletal and dental changes in the sagittal, vertical, and transverse dimensions after rapid palatal expansion. Am J Orthod Dentofacial Orthop 2004; 126: 569-75. [CrossRef]

14. Baratieri C, Alves M Jr, Bolognese AM, Nojima MC, Nojima LI. Changes in skeletal and dental relationship in Class II Division I malocclusion after rapid maxillary expansion: a prospective study. Dental Press J Orthod 2014; 19: 75-81. [CrossRef]

15. Lagravere MO, Major PW, Flores-Mir C. Long-term skeletal changes with rapid maxillary expansion: a systematic review. Angle Orthod 2005; 75: 1046-52.

16. da Silva Filho OG, Boas MC, Capelozza Filho L. Rapid maxillary expansion in the primary and mixed dentitions: a cephalometric evaluation. Am J Orthod Dentofacial Orthop 1991; 100: 171-9. [CrossRef]

17. Yilmaz BS, Kucukkeles N. Skeletal, soft tissue, and airway changes following the alternate maxillary expansions and constrictions protocol. Angle Orthod 2014; 84: 868-77.

18. Melsen B. The cranial base. Acta Odontol Scand 1974; 32.
19. Huang C, Wang Y, Huang C, Liou E. Maxillary displacement after rapid maxillary expansions: an animal study. J Taiwan Assoc Orthod 2008; 20: 19-31.

20. Canturk BH, Celikoglu M. Comparison of the effects of face mask treatment started simultaneously and after the completion of the alternate rapid maxillary expansion and constriction procedure. Angle Orthod 2015; 85: 284-91. [CrossRef]

21. Maino G, Turci Y, Arreghini A, Paoletto E, Siciliani G, Lombardo L. Skeletal and dentoalveolar effects of hybrid rapid palatal expansion and facemask treatment in growing skeletal Class III patients. Am J Orthod Dentofacial Orthop 2018; 153: 262-8. [CrossRef]

22. Haas AJ. Rapid expansion of the maxillary dental arch and nasal cavity by opening the mid-palate suture. Angle Orthod 1961; 31: 73-90.

23. Wertz RA. Skeletal and dental changes accompanying rapid midpalatal suture opening. Am J Orthod 1970; 58: 41-66. [CrossRef]

24. Sari Z, Uysal T, Usumez S, Basciftci FA. Rapid maxillary expansion. Is it better in the mixed or in the permanent dentition? Angle Orthod 2003; 73: 654-61.

25. Biederman W. Rapid correction of class III malocclusion by midpalatal expansion. Am J Orthod 1973; 63: 47-55. [CrossRef]

26. Davis WM, Kronman JH. Anatomical changes induced by splitting the midpalatal suture. Angle Orthod 1969; 39: 126-32.

27. Sarver DM, Johnston MW. Skeletal changes in vertical and anterior displacement of the maxilla with bonded rapid palatal expansion appliances. Am J Orthod Dentofacial Orthop 1989; 95: 462-6. [CrossRef]

28. Sung SJ, Baik HS. Assessment of skeletal and dental changes by maxillary protraction. Am J Orthod Dentofacial Orthop 1998; 114: 492-502. [CrossRef]

29. Cha KS. Skeletal changes of maxillary protraction in patients exhibiting skeletal Class III malocclusion: a comparison of three skeletal maturation groups. Angle Orthod 2003; 73: 26-35.

30. Kapust AJ, Sinclair PM, Turley PK. Cephalometric effects of face mask/ expansion therapy in Class III children: a comparison of three age groups. Am J Orthod Dentofacial Orthop 1998; 113: 204-12. [CrossRef] 